\title{
Gratings for High-Energy Petawatt Lasers
}

\section{UCRL-CONF-217007}

H. T. Nguyen, J. A. Britten, T. C. Carlson, J. D. Nissen, L. J. Summers, C. R. Hoaglan, M. D. Aasen, J. E. Peterson, I. Jovanovic

November 11, 2005

SPIE Boulder Damage Symposium XXXVII Boulder, CO, United States September 19, 2005 through September 23, 2005 
This document was prepared as an account of work sponsored by an agency of the United States Government. Neither the United States Government nor the University of California nor any of their employees, makes any warranty, express or implied, or assumes any legal liability or responsibility for the accuracy, completeness, or usefulness of any information, apparatus, product, or process disclosed, or represents that its use would not infringe privately owned rights. Reference herein to any specific commercial product, process, or service by trade name, trademark, manufacturer, or otherwise, does not necessarily constitute or imply its endorsement, recommendation, or favoring by the United States Government or the University of California. The views and opinions of authors expressed herein do not necessarily state or reflect those of the United States Government or the University of California, and shall not be used for advertising or product endorsement purposes. 


\title{
Gratings for High-Energy Petawatt Lasers
}

\author{
H.T. Nguyen, J.A. Britten, T.C. Carlson, J.D. Nissen, L.J. Summers, C.R. Hoaglan, M.D. Aasen, J.E. \\ Peterson, I. Jovanovic \\ Lawrence Livermore National Laboratory, P.O. Box 808, Livermore CA 94550
}

\begin{abstract}
To enable high-energy petawatt laser operation we have developed the processing methods and tooling that produced both the world's largest multilayer dielectric reflection grating and the world's highest laser damage resistant gratings. We have successfully delivered the first ever $80 \mathrm{~cm}$ aperture multilayer dielectric grating to LLNL's Titan Intense Short Pulse Laser Facility. We report on the design, fabrication and characterization of multilayer dielectric diffraction gratings.
\end{abstract}

Keywords: high-energy petawatt laser, multilayer dielectric diffraction grating, reactive ion beam etching.

\section{INTRODUCTION}

The technique of chirped pulse amplification ${ }^{1}$ (CPA) has enabled the generation of Petawatt-class laser system throughout the world. Existing Petawatt-class lasers today are based on Nd:glass architectures with laser pulses nominally of $500 \mathrm{~J}$ energy and pulse duration of $500 \mathrm{fs}$. These laser systems today use meter-scale aperture, goldovercoated master photoresist gratings to compress the final amplified chirped pulse $\mathrm{s}^{2,3,4}$. The realization of High-Energy Petawatt (HEPW) class laser systems, targeting laser pulses of greater $>1000 \mathrm{~J}$ energy and pulse durations in the 1-10 picosecond time regime, has been hindered by the limitation of the damage threshold of the gold-overcoated gratings. These limitations led to the development and successful demonstration of small aperture multilayer dielectric (MLD) gratings at Lawrence Livermore National Laboratory (LLNL) in the mid $90^{\text {' }} \mathrm{s}^{5,6}$.

\section{GRATING DESIGN}

The optical design of a high-efficiency MLD grating is subject to a number of constraints related to its manufacturability ${ }^{7}$. We choose to design a dichroic multilayer coating that is highly reflective at the use angle and wavelength, and minimally reflective at the holographic exposure angle and wavelength. This is to minimize 'reflective notching' common to pattern generation in photoresist on reflective structures, that impacts linewidth control. The second criterion in particular places demands on the accuracy of the coating deposition. We could choose to deposit a simpler quarter-wave design and use a sacrificial reflective coating between the multilayer stack and the photoresist film, but this increases complexity and risk for other aspects of the grating manufacturing process, particularly at large apertures. The design must also be insensitive to grating linewidth variations that can be expected to occur over the apertures considered here.

The grating can be etched into one or several of the deposited dielectric layers. We choose to have a single thick $\mathrm{SiO}_{2}$ layer comprising the grating, due to the intrinsic high laser damage threshold of $\mathrm{SiO}_{2}$ and the availability of robust designs incorporating this single layer. In general, our designs require deposition of two materials during the coating run. In the case of these demonstration gratings, the high-and low index layers were comprised of $\mathrm{HfO}_{2}$ and $\mathrm{SiO}_{2}$. The final design was the result of numerous iterations based on performance and deposition error-tolerance considerations. A typical desired grating design is shown in Figure 1. 

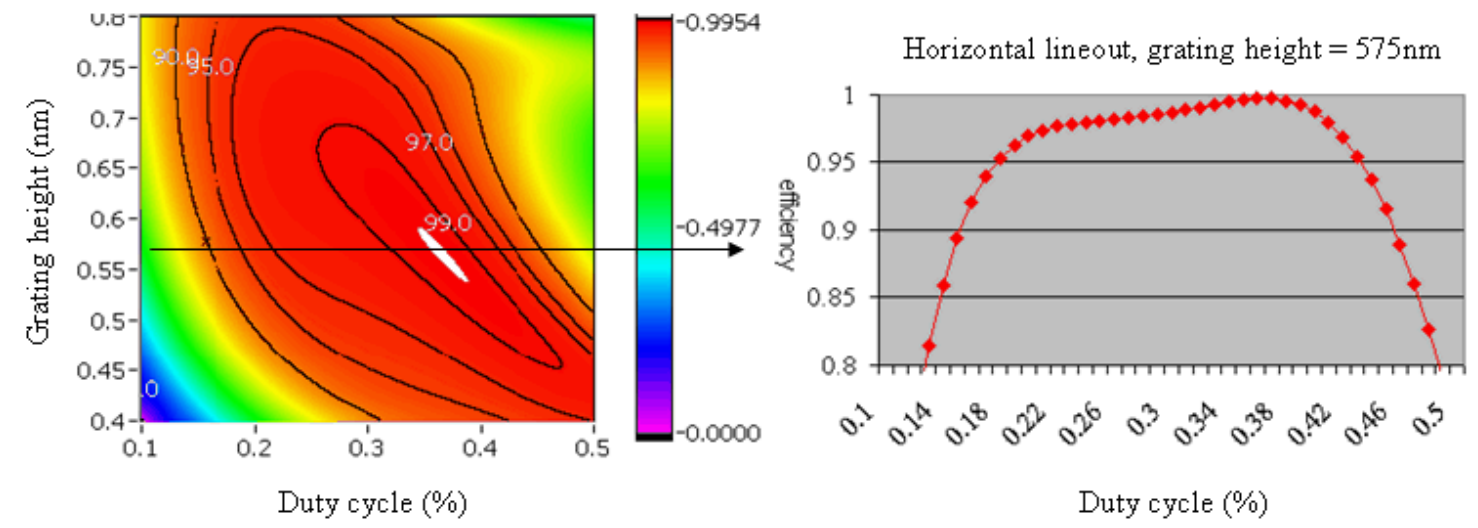

Figure 1: Predicted performance of a typical MLD grating where the high-and low index layers were comprised of $\mathrm{HfO}_{2}$ and $\mathrm{SiO}_{2}$. The duty cycle is define as width of the grating profile divide its period. The period in this particular case is $575 \mathrm{~nm}$.

\section{GRATING FABRICATION}

The fabrication of the MLD grating begins with a dielectric multilayer structure vacuum deposited onto an optically flat substrate. The substrate is then coated with a thin $(\approx 500 \mathrm{~nm})$ film of photoresist, using a meniscus coating process ${ }^{8}$, then baked in a convection oven overnight. The grating pattern was exposed into the photoresist layer using LLNL's large laser interference lithography station ${ }^{9}$, shown in Figure 2, utilizing 1.1 meter-diameter collimating lenses and $413 \mathrm{Kr}$-ion laser light. The surface relief pattern spacing $d$ is produced in the photoresist by intersecting two plane-wave laser beams, each of exposure $\lambda$ and each incident at an angle $\theta$ according to the following relationship,

$$
d=\frac{\lambda}{2 \sin \theta \cos \phi}
$$

where $\phi$ is the angle between the normal to the substrate and the bisector of the incident laser beams. Only highly collimated beams produce straight, parallel grooves; even the slightest wave-front curvature of the interfering beams produces curved grooves with non-uniform spacing. This groove distortion degrades the spectral resolution of the grating and produces curvature in the wave-front of the diffracted beam. After pattern development, the photoresist grating lines were examined at several locations using a custom large-bridge atomic force microscope capable of nondestructive measurement of submicron line widths anywhere on a $400 \times 800 \mathrm{~mm}$ aperture. These measurements verified the uniformity of the exposure/development process. The optics were then hard-baked to increase the resistance of the photoresist grating mask to the ion-beam etching process. 


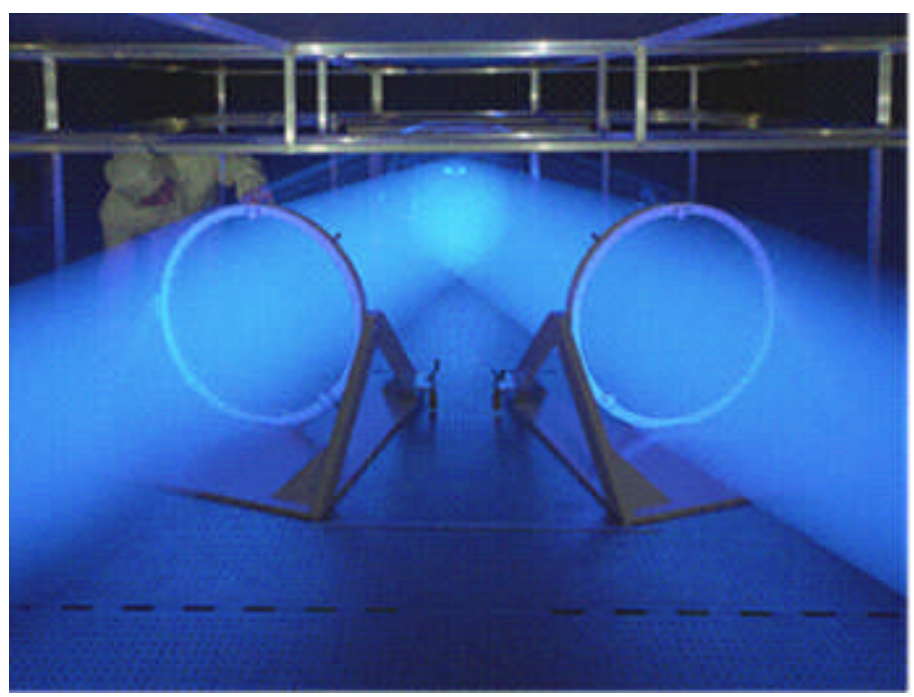

Figure 2: The exposure laser beams in the holographic exposure apparatus illuminate the $1100 \mathrm{~mm}$ aperture collimation lens.

The photoresist grating structure was transfer-etched into the top $\mathrm{SiO}_{2}$ layer of the grating using a custom reactive ionbeam etcher ${ }^{10}$, shown in Figure 3, capable of etching submicron features into substrates as large as $2000 \mathrm{x} 1000 \mathrm{~mm}$. This tool utilizes a linear 1.1-meter commercial RF-generated ion beam configured for reactive etching using a gas mix of $\mathrm{CHF}_{3}, \mathrm{Ar}$ and $\mathrm{O}_{2}$, for increased selectivity of oxide-to-resist etch rate. The ion source is optimized for uniform beam current, and is mounted vertically in the center of a long vacuum chamber. The substrate passes back and forth laterally across the beam at a constant rate, removing a few nm of material per pass until the desired etch depth is attained.

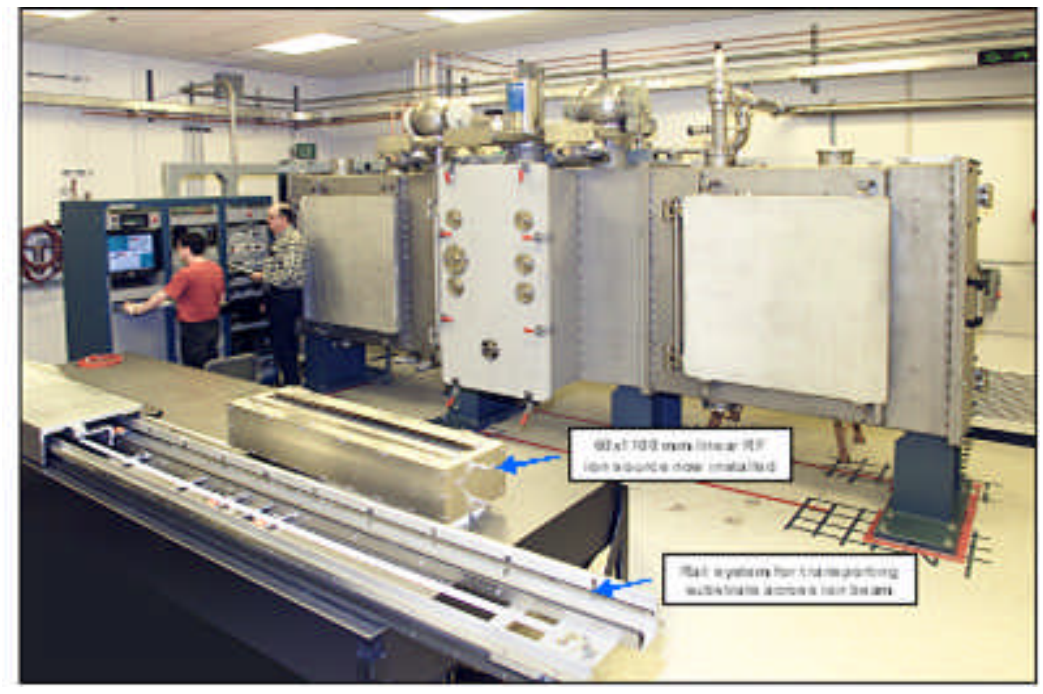

Figure 3: The world largest reactive ion beam system is capable of etching submicron features into substrates as large at $1000 \mathrm{x} 2000$ $\mathrm{mm}$.

After the etch process, the remaining photoresist mask was chemically stripped. The finished gratings were then subjected to full-aperture metrology including interferometry for diffracted and reflected wavefront, and photometry for the absolute efficiency in the -1 order. Witness parts processed from the same MLD coating batch as ride-alongs were cleaved and subjected to scanning electron microscopy. A cartoon of the complete fabrication steps is shown in Figure 4. 


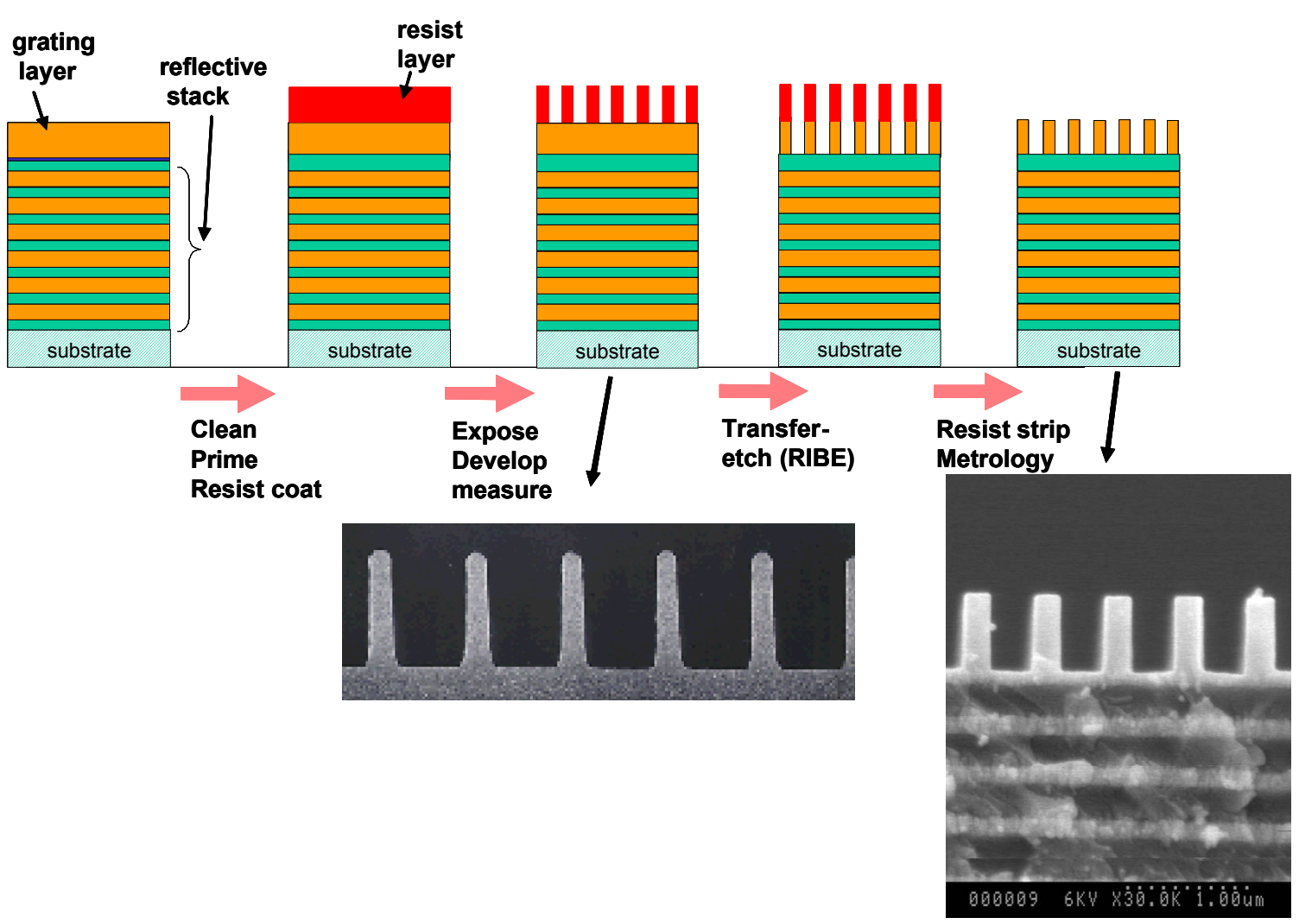

Figure 4: Process steps required in the fabrication of MLD gratings.

\section{DISCUSSION}

LLNL has successfully designed and fabricated the largest MLD grating to date, three $400 \times 800 \mathrm{~mm}$ size MLD gratings, $1780 \mathrm{l} / \mathrm{mm}$ for $1053 \mathrm{~nm}$ light, that exhibits $>96 \%,>96 \%$, and $>91 \%$ diffraction efficiency respectively. The RMS diffraction efficiency uniformity was measured to be less than $1.5 \%$ over the full aperture for gratings. A laser photometry map of one of the grating's diffraction efficiency is shown in Figure 5. Just as importantly, with new MLD grating designs and processing technology, LLNL has been able to increase the laser damage threshold of these optics by a factor of 4-5 compared to gold over-coated gratings at $10 \mathrm{ps}$. Conditioned laser damage thresholds as high as $4.5 \mathrm{~J} / \mathrm{cm}^{2}$ have been measured at our lab for $10 \mathrm{ps}$ pulses at TE polarization and $76.5^{\circ}$ incidence angle on $1780 \mathrm{l} / \mathrm{mm}$ MLD gratings. These are the highest measured value to date for a MLD grating of any size. A photo of a $400 \times 800 \mathrm{~mm}$ MLD grating is shown in Figure 6. 


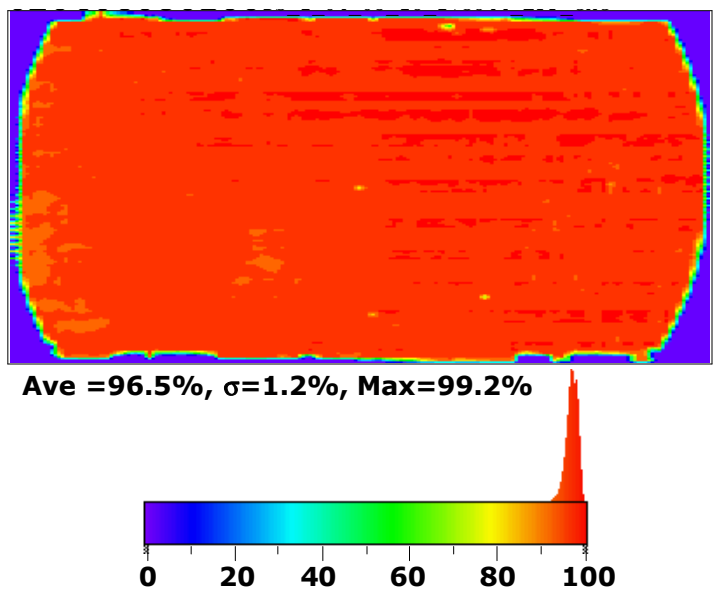

Figure 5: Diffraction efficiency map and histogram of a $800 \mathrm{~mm}$ diameter (truncated to $400 \mathrm{~mm}$ vertical dimension) MLD grating at $1780 \mathrm{l} / \mathrm{mm}$ shows excellent uniformity of diffraction efficiency over the entire aperture for $1053 \mathrm{~nm}$, TE polarization at $66^{\circ}$ incidence angle.

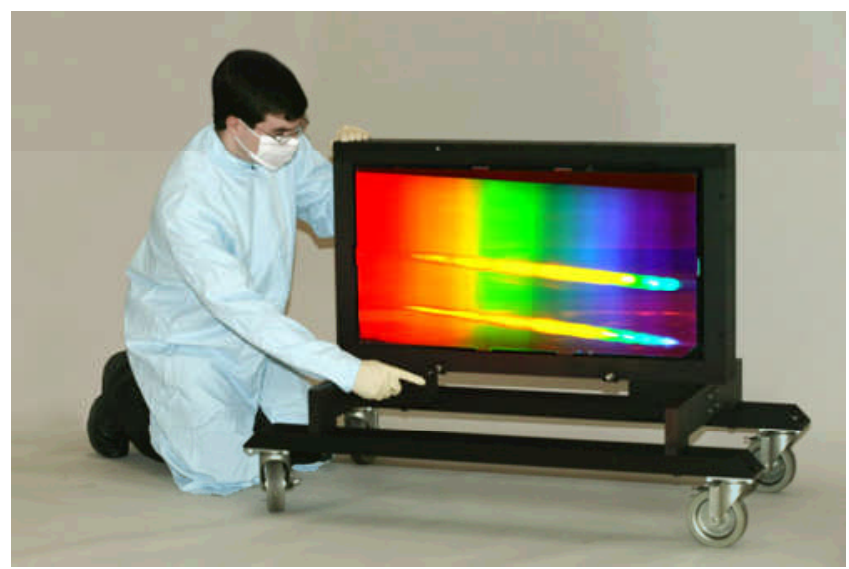

Figure 6: LLNL engineer inspects a 400 x $800 \mathrm{~mm}, 1780$ 1/mm MLD grating designed for use at $1053 \mathrm{~nm}$, TE

The grating was further examined by full-aperture interferometry using a Zygo 18"-aperture, phase-shifting interferometer operating at $1064 \mathrm{~nm}$ at both ambient conditions and in a dry nitrogen-purged environment to simulate the wavefront alteration under use conditions in a vacuum. The diffracted wavefront was measured in the Littrow condition $\left(71.2^{\circ} @ 1064 \mathrm{~nm}\right.$ ) for both orientations: grating surface normal rotated $\mathrm{CW}(\rightarrow /)$ and $\mathrm{CCW}(\rightarrow \mid)$ with respect to the incoming beam. The zero-order reflected wavefront was also measured. Figure 7 shows the diffracted wavefront in the $\rightarrow \backslash$ orientation under dry conditions. The overall PV of 0.255 waves is considered to be extremely good for an optic of this size and shape that has both surface figure and holographic errors. Table 1 summarizes interferometric data of all of the measurements. The zero order wavefront and the $\rightarrow$ / diffracted order wavefront are dominated by convex astigmatism in the long axis. The convex cylinder error in the reflected wavefront is due to residual compressive stress in the multilayer coating. It appears that in the $\rightarrow \backslash$ orientation astigmatism from holographic error largely cancels the coated surface figure error, while in the $\rightarrow$ / orientation these errors add to worsen the diffracted wavefront. Of particular note is the very minor change in wavefront exhibited between ambient and dry environments. This is atypical of standard multilayer coatings manufactured at the University Rochester Laboratory for Laser Energetics (LLE) with electron-beam deposition. The majority of NIF manufactured coatings show a change in stress of approximately $40 \mathrm{MPa}$ between ambient (nominally $40 \% \mathrm{RH}$ ) and dry conditions. Often, exposure to a dry environment causes water loss in porous e-beam evaporated coatings, resulting in the buildup of tensile stresses. In extreme cases, the coating will crack or craze due to these stresses. LLE deposition processes are optimized for neutral stress for the specific use environment of a coating. In this particular case, there was little change in stress between the ambient and dry environments. This requires a closer review of the MLD coating design where the stress contributions from each material balance the overall shift. The residual compressive stress of this coating, even after etching through the thick $\mathrm{SiO}_{2}$ top layer and exposure to dry conditions, ensures that the coating will not craze under use conditions. 


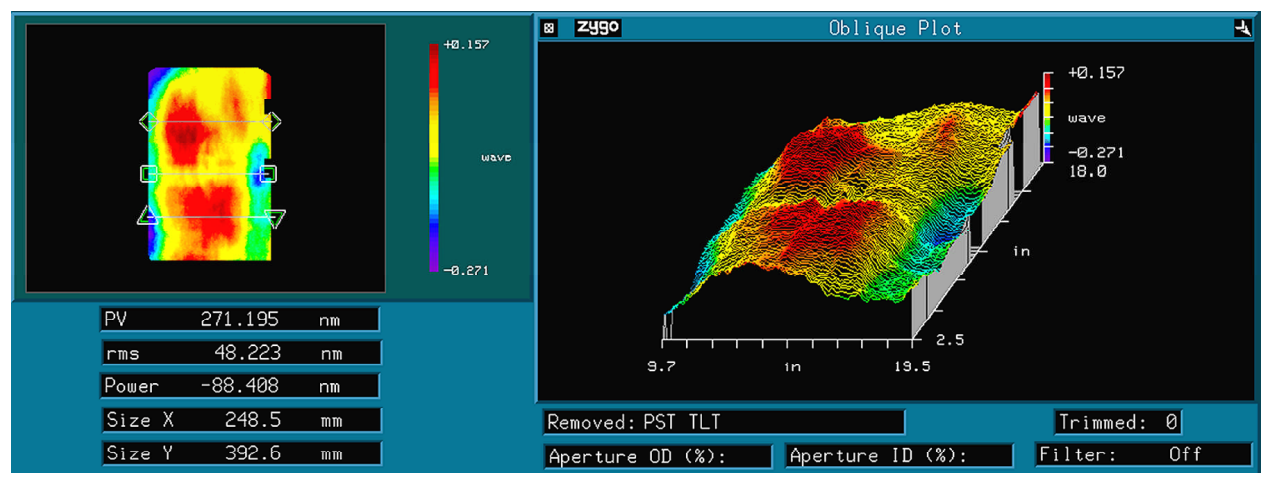

Figure 7: Diffracted wavefront of grating of Figure 5 at $1053 \mathrm{~nm}$, at Littrow angle of $71.2^{\circ}$.

Table 1: Interferometry results taken at LLE for $800 \mathrm{x} 400 \mathrm{~mm}$ grating measured at $1064 \mathrm{~nm}$, Littrow angle $\left(71.2^{\circ}\right)$. Data are for reflected and diffracted wavefront, measured in waves at $1064 \mathrm{~nm} . \rightarrow /$ and $\rightarrow \backslash$ represent orientation of the grating with respect to the incoming light.

\begin{tabular}{|l|c|c|c|c|}
\cline { 2 - 5 } \multicolumn{1}{c|}{} & \multicolumn{2}{c|}{ Ambient } & \multicolumn{2}{c|}{ Dry } \\
\hline $\begin{array}{c}\text { reflected/ diffracted } \\
\text { wavefront }\end{array}$ & $\begin{array}{c}\text { P-V Waves @ } \\
\mathbf{1 0 6 4} \mathbf{~ n m}\end{array}$ & $\begin{array}{c}\text { RMS Waves @ } \\
\mathbf{1 0 6 4} \mathbf{~ n m}\end{array}$ & $\begin{array}{c}\text { P-V Waves @ } \\
\mathbf{1 0 6 4} \mathbf{~ n m}\end{array}$ & $\begin{array}{c}\text { RMS Waves @ } \\
\mathbf{1 0 6 4} \mathbf{~ n m}\end{array}$ \\
\hline-1 order $\rightarrow /^{*}$ & 0.654 & 0.121 & 0.706 & 0.151 \\
-1 order $\rightarrow \backslash$ & 0.276 & 0.039 & 0.255 & 0.045 \\
0 order & 0.261 & 0.063 & -- & -- \\
\hline
\end{tabular}

*Dominated by convex astigmatism along long axis.

\section{CONCLUSION}

LLNL has designed, constructed, and implemented a world-class facility to manufacture $\sim 1$ meter size MLD grating. MLD gratings are essential for the realization of High-Energy Petawatt class laser systems here at LLNL and around the world. With the availability of $\sim 1$ meter scale MLD gratings, HEPW systems like LLNL's Janus ( $>400 \mathrm{~J}$ in $400 \mathrm{fs}$ ), LLNL's NIF ARC (>1000 J in $10 \mathrm{ps})$, others.......can be realized. LLNL will continue to further its efforts in the design and fabrication of MLD gratings with a goal of manufacturing $\sim 1$ meter-scale optics with $>99 \%$ diffraction efficiency over the entire aperture and a laser damage threshold in excess of $4 \mathrm{~J} / \mathrm{cm}^{2}$.

This work was performed under the auspices of the United States Department of Energy by University of California, Lawrence Livermore National Laboratory under contract no. W-7405-Eng-48.

\section{REFERENCES}

1. D. Strickland and G. Mourou, 'Compression of Amplified Chirped Optical Pulses', Opt. Commun., 56, 219-221, (1985)

2. M.D. Perry, D. Pennington, B.C. Stuart, G. Tietbohl, J.A. Britten, C. Brown, S. Herman, B. Golick, M. Kartz, J. Miller, H. T. Powell, M. Vergino, V. Yanovsky, "Petawatt laser pulses" Optics Letters, 24, 160-162, (1999)

3. Kitagawa, Y., Fujita, H. Kodama, R., Yoshida, H., Matsuo, S, Jitsuno, T., Kawasaki, T., Kitamura, H., Kanabe, T., Sakabe, S., Shigemori, K., Miyanaga, N., Izawa, Y., "Prepulse-free PetaWatt laser for a fast ignitor" IEEE $J$. Quantum Electronics, 40,281_293, (2004)

4. M.H.R Hutchinson, D. Neely, B.E. Wyborn, 'Vulcan Upgrade: a PetaWatt laser facility for experiments at $10^{21} \mathrm{~W}$ $\mathrm{cm}^{-2}$, Proc. SPIE, V4424, 63-69, (2001).

5. M.D. Perry, J.A. Britten, H.T. Nguyen, R.D. Boyd, B.W. Shore, 'Multilayer Dielectric Diffraction Gratings', U.S. Patent no. 5907436, (1999) 
6. M.D. Perry, R.D. Boyd, J.A. Britten, D. Decker, B.W. Shore, C. Shannon, E. Shults, and L. Li, 'High Efficiency Multilayer Dielectric Diffraction Gratings', Optics Letters, 20(8) 940-942, (1995)

7. B.W. Shore, M.D. Perry, J.A. Britten, R.D. Boyd, M.D. Feit, H. T. Nguyen, R. Chow, G. Loomis and L. Li, 'Design of high-efficiency dielectric reflection gratings' Journal of the Optical Society of America A, 14(5), 1124-1136, (1997)

8. J.A. Britten, 'A Simple Theory for the Entrained Film Thickness During Meniscus Coating' Chemical Engineering Communications 120 59-71, (1993)

9. J.A. Britten, R.D. Boyd and B.W. Shore, 'In-situ Endpoint Detection During Development of Submicron Grating Structures in Photoresist', Optical Engineering 34(2) 474-479, (1995)

10. J.A. Britten, L.M. Jones II, T.C. Carlson, S.J. Bryan, C.R. Hoaglan, L.M. Risinger, A.C. Iyer, G.K.Beer, C.P.J. Barty, 'Enabling technology for fabrication of meter-scale gratings for high-energy Petawatt lasers"', (2004)

*hnguyen@llnl.gov; phone 1925423 6270; fax 1925424 6333; llnl.gov 